\title{
Contribución al conocimiento de la diversidad y conservación de los mamíferos en la cuenca del río Apurímac, Perú
}

\section{Contribution to the diversity and conservation knowledge of mammals in the basin of the Apurimac river, Peru}

\author{
Víctor Pacheco ${ }^{1,2}$, Edith Salas', Lianka Cairampoma1', Maggie Noblecilla1, Heidi \\ Quintana ${ }^{1}$, Floro Ortiz ${ }^{1}$, Pedro Palermo ${ }^{1}$ y Roberto Ledesma ${ }^{1}$
}

${ }^{1}$ Museo de Historia Natural, Universidad Nacional Mayor de San Marcos, Apartado 14-0434, Lima14, Perú.

${ }^{2}$ Facultad de Ciencias Biológicas, Universidad Nacional Mayor de San Marcos.

Email: Victor Pacheco vpachecot@unmsm.edu.pe
Presentado: $\quad 12 / 02 / 2007$ Aceptado: $\quad 22 / 11 / 2007$

\section{Resumen}

El presente trabajo documenta la diversidad de los mamíferos de la cuenca del río Apurímac, uno de los vacíos de información más importantes del Perú, en base a una evaluación de cinco lugares de muestreo en los departamentos de Apurímac (Cconoc, Velavelayoc), Ayacucho (Yanamonte, Ccentabamba) y Cusco (Catarata). El esfuerzo de captura fue de 1280 trampas noche y 41 redes noche. Se registraron 60 especies en el área de estudio; incluyendo 15 especies bajo alguna categoría de conservación y cuatro endémicas a nivel del país. Como era de esperarse, los murciélagos y los roedores fueron los órdenes más diversos $(66,7 \%)$. La diversidad de especies en los sitios de muestreo decrece significativamente con la elevación desde los puntos de muestreo más bajos (Ccentabamba y Catarata) hasta el más alto (Velavelayoc). El marsupial Monodelphis peruviana y el venado enano Mazama chunyi son primeros registros para el departamento de Ayacucho. Los murciélagos Artibeus planirostris y Myotis keaysi así como la nutria Lontra longicaudis son primeros registros para el departamento de Apurímac. La abundancia relativa presentó una correlación negativa significativa con la elevación para los murciélagos pero no fue significativa para los mamíferos pequeños terrestres. Las especies de Sturnira, Akodon y Thomasomys fueron las que obtuvieron una mayor abundancia relativa. Los índices de diversidad de Shannon-Wiener y Simpson mostraron también una correlación negativa significativa con la elevación. Se estima que la cuenca del río Apurímac alberga al menos 97 especies de mamíferos lo que resulta de compilar nuestros resultados con publicaciones previas. Se recomienda establecer áreas de conservación en Huanipaca, Yanamonte y Catarata e incrementar el área del Santuario Histórico Machu Picchu hasta incluir la zona del complejo arqueológico Choquequirao.

Palabras clave: Mamíferos, Perú, río Apurímac, diversidad, conservación, bosques montanos, VRAE.

\section{Abstract}

The present work documents the diversity of mammals in the headwaters of the Apurímac river, one of the most important information gaps in Peru, based on an evaluation of five sampling areas in the departments of Apurímac (Cconoc, Velavelayoc), Ayacucho (Yanamonte, Ccentabamba) and Cuzco (Catarata). Capture's effort was 1280 trap-nights and 41 mistnet-nights. Sixty species were recorded in the study area and included 15 threatened species and four species endemic to Perú. As expected, bats and rodents were the most diverse orders $(66,7 \%)$. The species diversity in the sites sampled declines significantly with elevation from the lowest elevation sites (Ccentabamba and Catarata) to the highest (Velavelayoc). The marsupial Monodelphis peruviana and the Dwarf Brocket deer Mazama chunyi are first records for the department of Ayacucho. The bats Artibeus planirostris and Myotis keaysi, and the river otter Lontra longicaudis are first records for the department of Apurímac. For bats, the relative abundance showed a significant negative correlation with elevation, but was not significant for small terrestrial mammals. The species of Sturnira, Akodon and Thomasomys had the highest values of relative abundance. The diversity indices (Shannon-Wiener and Simpson) also showed also a significant negative correlation with elevation. The Apurímac river headwater is estimated to have at least 97 mammals species. We recommend creating conservation areas in Huanipaca, Yanamonte and Catarata, and enlarging the area of the Santuario Histórico Machu Picchu to include the archaeological site of Choquequirao.

Keywords: Mammals, Peru, Río Apurímac, diversity, conservation, montane forests, VRAE.

\section{Introducción}

El Perú tiene una gran diversidad de especies de mamíferos, estimándose esta diversidad en por lo menos 499 especies ( $\mathrm{Pa}$ checo, com. pers.), por lo que es considerado entre los países con mayor riqueza de especies a nivel mundial (Pacheco, 2002). Esta diversidad varía según la región biogeográfica del país, con la mayor diversidad de especies en los bosques bajos tropicales (Voss y Emmons, 1996; Emmons y Feer, 1997; Pacheco, 2002), seguido por las Yungas y otras regiones biogeográficas (Pacheco, 2002).
Una de las zonas poco conocidas, en su diversidad de mamíferos, es la cuenca del río Apurímac, componente principal de la región denominada VRAE (Valle del río Apurímac y Ene). El río Apurímac nace en el nevado Mismi en el departamento de Arequipa y corre en dirección SE-NO por los departamentos de Cusco, Apurímac y Ayacucho, formando un profundo talud, en tal grado que es considerado el río más profundo del Perú, de América, y posiblemente del Mundo. Este río tiene como tributarios importantes a los ríos Pampas, Pachachaca y Mantaro y forma a lo largo de su recorrido una amplia varie- 


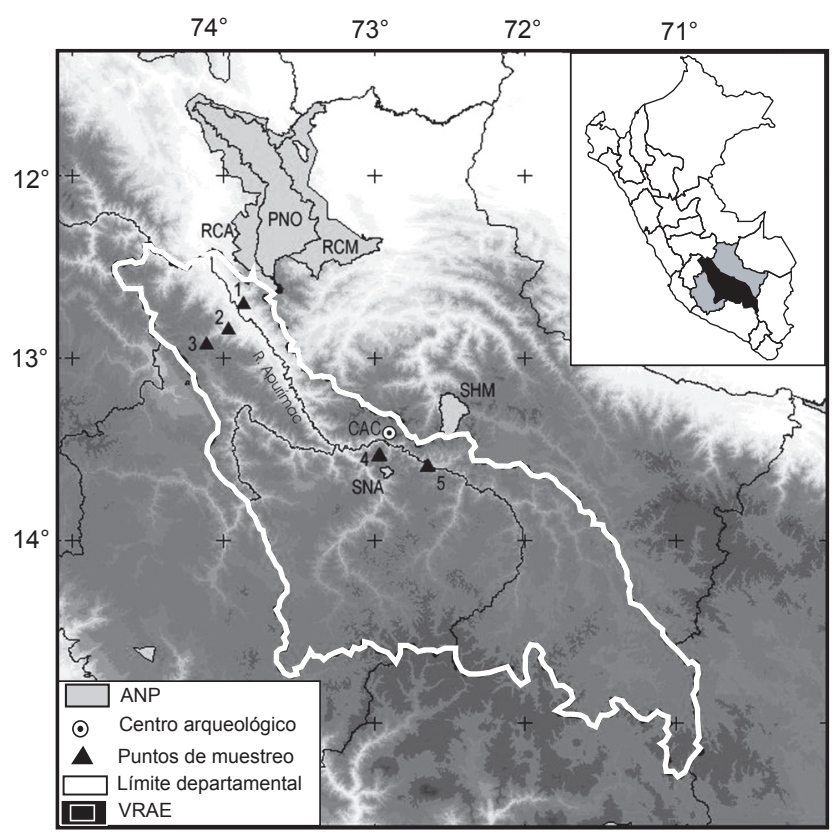

Figura 1. Mapa de las localidades evaluadas en la cuenca del río Apurímac: 1 = Catarata, $2=$ Ccentabamba, $3=$ Yanamonte, 4 = Velavelayoc, $5=$ Cconoc. $\mathrm{RCA}=$ Reserva Comunal Ashaninka, $\mathrm{PNO}=$ Parque Nacional Otishi, RCM = Reserva Comunal Machiguenga, SHM = Santuario Histórico Machupicchu, SNA = Santuario Nacional Ampay, CAC = Centro Arqueológico Choquequirao.

dad de ambientes, siendo el matorral arbustivo, el bosque seco interandino, el bosque premontano tropical, la yunga y la puna los más conspicuos.

El terrorismo ocurrido en la década de 1980 fue el principal factor que impidió el acceso a la zona y por ende el desarrollo de estudios científicos. Previo a estos sucesos se realizaron las expediciones a la Cordillera de Vilcabamba por J.W. Terborgh y J. S. Weske de 1966 al 1972 (ver Terborgh y Dudley, 1973), cuyos resultados fueron presentados en su mayor parte por Koopman (1978); las expediciones a la cuenca del río Apurímac por J.W. Terborgh, J. S. Weske, A.L. Gardner y J.P. O’Neill entre 1967 y 1971, cuyos resultados están publicados en Koopman (1978), Gardner y O’Neill (1971) y Gardner y Patton (1972); y los estudios sobre el oso de anteojos por Peyton (1980). Después de que el terrorismo cesa en la zona se publican trabajos relacionados a las exploraciones del proyecto Camisea como el de Solari et al. (2001a, b) y Rodríguez y Amanzo (2001); mientras que otros enfatizan aspectos sistemáticos y de distribución de especies presentes en la zona, como los de Pacheco (2003), Pacheco et al. (2004), Velazco (2005) y Pacheco y Hocking (2006) entre otros.

El presente trabajo contribuye a documentar la diversidad de mamíferos para la cuenca del río Apurímac en base a trabajos de campo realizados en los departamentos de Apurímac, Ayacucho y Cusco, revisión de bases de datos y literatura especializada, con los objetivos de: 1) Producir una lista de especies presentes para el área de estudio, 2) Determinar la abundancia relativa de las especies registradas, 3) Evaluar el estado de conservación de las especies endémicas, raras, o listadas en alguna categoría de conservación y 4) Sugerir la conservación de algunas áreas basadas en criterios de diversidad, endemismo y presencia de especies amenazadas.

\section{Área de estudio:}

La cuenca del río Apurímac (Fig. 1) es una región muy extensa, de aproximadamente $27548.06 \mathrm{~km}^{2}$, siendo más grande que varios departamentos del Perú y equiparable en área al departamento de Pasco. Cinco lugares de muestreo presentes en dicha cuenca fueron evaluados en el 2006: Cconoc y Velavelayoc en el departamento de Apurímac (16 al 23 de Marzo), Yanamonte y Ccentabamba en el departamento de Ayacucho (17 al 24 de Mayo) y Catarata en el departamento de Cusco (25 al 28 de Mayo). La localidad, coordenadas y elevación se detallan en la Tabla 1 .

Cconoc: Departamento de Apurímac. El lugar de muestreo se ubica en la margen izquierda y al borde del río Apurímac, en los alrededores del albergue turístico Cconoc. Vegetación de bosque seco interandino de algarrobo, huarango y azafrán, algunos árboles muy altos de hasta $15 \mathrm{~m}$ y cerca de $1 \mathrm{~m}$ de diámetro, formando parches de bosques o dispersos. Suelo pedregoso y rocoso, relativamente plano, con zonas arenosas y arcillosas en el margen del río.

Velavelayoc: Departamento de Apurímac. El lugar de muestreo se ubica al norte y a dos horas de caminata de Huanipaca. Vegetación de bosque montano primario húmedo y de matorral de arbustos. El primero con una cobertura vegetal de $90 \%$, con árboles de 4 a $5 \mathrm{~m}$ en promedio, sobre los que se encuentran musgos, líquenes, bromeliáceas y helechos epifitos. El hábitat de matorral de arbustos es denso y espinoso, con pocos arbolillos que se hacen más densos al acercarse a una quebrada.

Tabla 1. Ubicación de los lugares de muestreo en la cuenca del río Apurímac.

\begin{tabular}{|c|c|c|c|c|c|}
\hline \multirow{2}{*}{ Localidad } & \multirow{2}{*}{$\begin{array}{l}\text { Unidad de } \\
\text { vegetación }\end{array}$} & \multirow{2}{*}{ Transectos } & \multirow{2}{*}{$\begin{array}{l}\text { Altitud } \\
\text { (msnm) }\end{array}$} & \multicolumn{2}{|c|}{ Coordenadas } \\
\hline & & & & Sur & Oeste \\
\hline \multirow{6}{*}{ Velavelayoc } & \multirow{4}{*}{$\begin{array}{l}\text { Bosque } \\
\text { montano } \\
\text { andino }\end{array}$} & Apu-06 & \multirow{2}{*}{3500} & \multirow{2}{*}{-13.4758} & \multirow{2}{*}{-72.9368} \\
\hline & & Apu-11 & & & \\
\hline & & Apu-05 & \multirow{2}{*}{3450} & \multirow{2}{*}{-13.4751} & \multirow{2}{*}{-72.9287} \\
\hline & & Apu-10 & & & \\
\hline & \multirow{2}{*}{$\begin{array}{l}\text { Matorral de } \\
\text { arbustos }\end{array}$} & Apu-04 & \multirow{2}{*}{3450} & \multirow{2}{*}{-13.4733} & \multirow{2}{*}{-72.9286} \\
\hline & & Apu-09 & & & \\
\hline \multirow{5}{*}{ Yanamonte } & \multirow{5}{*}{$\begin{array}{l}\text { Bosque } \\
\text { montano }\end{array}$} & Apu-12 & \multirow{5}{*}{2751} & \multirow{5}{*}{-12.785} & \multirow{5}{*}{-73.995} \\
\hline & & Apu-13 & & & \\
\hline & & Apu-14 & & & \\
\hline & & Apu-19 & & & \\
\hline & & Apu-20 & & & \\
\hline \multirow{5}{*}{ Cconoc } & \multirow{5}{*}{$\begin{array}{l}\text { Bosque seco } \\
\text { interandino }\end{array}$} & Apu-01 & \multirow{2}{*}{1819} & \multirow{2}{*}{-13.5428} & \multirow{2}{*}{-72.6358} \\
\hline & & Apu-07 & & & \\
\hline & & Apu-02 & 1819 & -13.5425 & -72.634 \\
\hline & & Apu-03 & \multirow{2}{*}{1822} & \multirow{2}{*}{-13.5394} & \multirow{2}{*}{-72.6436} \\
\hline & & Apu-08 & & & \\
\hline \multirow{3}{*}{ Ccentabamba } & \multirow{3}{*}{$\begin{array}{l}\text { Bosque } \\
\text { premontano } \\
\text { alto }\end{array}$} & Apu-15 & \multirow{3}{*}{1050} & \multirow{3}{*}{-12.6944} & \\
\hline & & Apu-16 & & & -73.8633 \\
\hline & & Apu-21 & & & \\
\hline & Bosque & Apu-17 & & & \\
\hline Catarata & premontano & Apu-18 & 805 & -12.5363 & -73.7703 \\
\hline & bajo & Apu-22 & & & \\
\hline
\end{tabular}


Yanamonte: Departamento de Ayacucho. El lugar de muestreo se ubica arriba de Ccano y al lado derecho de la carretera de Tambo a Ccano. Vegetación de bosque montano compuesta por arbustos grandes, chusqueas y árboles medianos de 5 a 10 $\mathrm{m}$ de altura; presenta zonas de purmas y "chacras" (campos de cultivo) de maíz, calabazas y otras hortalizas. Suelo pedregoso con pendiente moderada.

Ccentabamba: Departamento de Ayacucho. El lugar de muestreo se ubica al margen derecho de la carretera Ccentabamba a San Francisco, a lo largo de una quebrada. Vegetación de bosque premontano compuesta por árboles de hasta $15 \mathrm{~m}$ aprox. y arbustos medianos. Presenta también grandes áreas de chacras de café, cacao y naranja. Suelo con pendiente fuerte.

Catarata: Departamento de Cusco. El lugar de muestreo se ubica cerca al pueblo Catarata y al pie de un gran risco con tres cataratas. Vegetación con parches de bosque premontano, donde los árboles alcanzan una altura de 20 a 25 m aprox. con sotobosque descubierto. Presenta también grandes áreas de chacra de café, cacao, pińa, naranja, limón y coca. Suelo pedregoso y pendiente moderada a fuerte.

\section{Material y métodos}

La metodología para el estudio de los mamíferos es muy diversa, pero el empleado aquí es una modificación de los métodos encontrados en Jones et al. (1996), Voss y Emmons (1996) y Woodman et al. (1996). Los mamíferos pequeños terrestres fueron evaluados usando trampas de golpe (Víctor rat trap), Museum Special y trampas de caja (Sherman live traps). Los dos primeros tipos de trampas sacrifican a los animales al capturarlos, mientras que el último tipo los captura vivos. Los especímenes capturados vivos se sacrificaron mediante dislocación cervical. Las trampas fueron dispuestas en transectos de 30 estaciones, con un par de trampas por estación, por lo general una combinación de Víctor-Sherman o Museum Special-Sherman. Se procuró una separación de aproximadamente $10 \mathrm{~m}$ (o 15 pasos) entre las estaciones de trampeo, formando una línea recta en lo posible y en un hábitat relativamente homogéneo. Las trampas fueron cebadas con una mezcla de avena, mantequilla de maní, vainilla, miel de abeja y diferentes tipos de semillas para canarios, en una proporción aproximada de 10:3:1:1:1; ocasionalmente un plátano maduro fue incluido en la mezcla. Las trampas fueron armadas en la tarde, revisadas en las primeras horas de la mañana y cebadas nuevamente en la tarde.

Para la evaluación de murciélagos se utilizaron redes de niebla de $12 \mathrm{~m}$ o $6 \mathrm{~m}$ de largo por 2,5 $\mathrm{m}$ de alto dispuestas en lugares óptimos, como caminos o quebradas, cerca de posibles refugios (e.g., cuevas, acantilados) y áreas de forrajeo (e.g., cerca de árboles con frutos, humedales, o estanques). Las redes fueron revisadas cada hora aproximadamente, entre las 18:00 y las 24:00; cerrándose en la última revisión. Algunas redes permanecieron ocasionalmente abiertas hasta el amanecer del día siguiente.

Debido a la gran extensión del área de estudio y para obtener una mejor representatividad, los transectos fueron establecidos en diferentes tipos de hábitats, moviéndose éstos frecuentemente, favoreciéndose una aproximación extensiva en lugar de una intensiva. Se establecieron en total 13 transectos para la captura de mamíferos terrestres no voladores, y 10 transectos para voladores (ver Tablas 1,2).

Los especímenes capturados se preservaron como pieles, cráneos y carcasas, o ejemplares completos en líquido (fijado en
Tabla 2. Esfuerzo de captura de pequeños mamíferos terrestres y voladores por localidad evaluada en la cuenca del río Apurímac.

\begin{tabular}{cccccc}
\hline & & \multicolumn{2}{c}{ Trampas noche } & \multicolumn{2}{c}{ Redes noche } \\
\cline { 3 - 6 } Localidad & $\begin{array}{c}\text { Unidad de } \\
\text { vegetación }\end{array}$ & $\begin{array}{c}\text { N. }{ }^{\circ} \text { de } \\
\text { transectos }\end{array}$ & $\begin{array}{c}\text { Esfuerzo } \\
\text { de } \\
\text { captura }\end{array}$ & $\begin{array}{c}\text { N. }{ }^{\circ} \text { de } \\
\text { transectos }\end{array}$ & $\begin{array}{c}\text { Esfuerzo } \\
\text { de } \\
\text { captura }\end{array}$ \\
\hline Velavelayoc & BMA, MA & 3 & 230 & 3 & 6 \\
Yanamonte & BM & 3 & 280 & 2 & 15 \\
Cconoc & BS & 3 & 420 & 3 & 6 \\
Ccentabamba & BPA & 2 & 180 & 1 & 5 \\
Catarata & BPB & 2 & 170 & 1 & 9 \\
Total & & 13 & 1280 & 10 & 41 \\
\hline
\end{tabular}

$\mathrm{BMA}=$ Bosque montano andino, $\mathrm{MA}=$ Matorral de arbustos, $\mathrm{BM}=$ Bosque montano, BS = Bosque seco interandino, $\mathrm{BPA}=$ Bosque premontano alto, BPB = Bosque premontano bajo

formol al 10\% por 10 días y luego preservado en alcohol al 70\%), y fueron identificados con un número de colector. Se registraron los datos del lugar de muestreo, tipo de hábitat, la estación y tipo de trampa. Adicionalmente, se tomaron las medidas estándar (longitud total, de la cola, del pie y oreja; y del antebrazo y el trago para los murciélagos), el peso, sexo, edad y la condición reproductiva de cada espécimen colectado. Se preservaron un mínimo de ejemplares por especie (generalmente tres) y por sitio de colecta para documentar la diversidad, liberándose los demás, registrándose previamente sus medidas, peso, sexo, hábitats de colecta y tomándose fotografías. Los especímenes se encuentran depositados en el Museo de Historia Natural de la Universidad Nacional Mayor de San Marcos, Lima-Perú, para cualquier referencia o estudio posterior.

La presencia de los mamíferos de mayor tamaño fue evaluada mediante entrevistas y registros directos (observaciones, vocalizaciones, etc.) e indirectos (huellas, heces, madrigueras, etc.). Se entrevistó a pobladores o trabajadores locales, preferentemente varones residentes en el área de estudio, con ayuda de un modelo de preguntas preestablecidas e ilustraciones de especies probablemente presentes en la zona (en: Emmons y Feer 1997). Las preguntas se desarrollaron de una forma tal en que se solicita que el encuestado describa lo que sabe o ha visto, en lugar de pedirle confirme lo que el entrevistador conoce. Por ejemplo, se pregunta simplemente cómo son las astas del venado que ha visto, en lugar de preguntarle si las astas tenían dos o muchas ramas. La información dudosa, conflictiva o presumiblemente falsa fue evaluada luego de la entrevista, nunca durante esta. Las observaciones registradas en los lugares de muestreo se realizaron durante el establecimiento o la revisión de los transectos, o durante el desplazamiento entre localidades, anotándose para cada observación la hora, ubicación (GPS), tipo de vegetación y número de individuos. Las especies registradas a través de encuestas u observaciones no fueron incluidas en los análisis cuantitativos.

Se sigue la nomenclatura taxonómica empleada en Wilson y Reeder (2005), excepto por los siguientes cambios o adiciones recientes: Artibeus planirostris diferenciado de A. jamaicensis por Lim et al. (2004); Carollia benkeithi recientemente reportada por Solari y Baker (2006) y diferenciada de C. castanea; Carollia manu, especie descrita por Pacheco et al. (2004); Monodelphis peruviana 
es aceptada como especie diferente por Solari (2007); los géneros Nephelomys, Euryoryzomys y Hylaemays recientemente separados del género Oryzomys por Weksler et al. (2006), formándose las combinaciones nuevas Nephelomys keaysi, Euryoryzomys nitidus, Hylaemays perenensis y H. yunganus; Platyrrhinus albericoi y $P$. masu descritas y diferenciadas de $P$. dorsalis por Velazco (2005), quien reconoce también a $P$. nigellus para las poblaciones peruanas en lugar de $P$. lineatus. Provisionalmente se sigue la propuesta de reconocer Odocoileus peruvianus por Molina y Molinari (1999) basado en un análisis morfométrico. Los nombres comunes empleados siguen mayormente a Pacheco et al. (1995).

Para estandarizar el esfuerzo de captura, este se expresa como trampas noche para el caso de los pequeños mamíferos terrestres y como redes noche para los mamíferos voladores. Se determina la abundancia relativa de una especie en relación al esfuerzo de captura empleado. Así, la abundancia relativa de los marsupiales y roedores es el número de individuos capturados (incluyendo los liberados) por cada 100 trampas noche, y el de murciélagos es el número de individuos capturados (incluyendo los liberados) por cada 10 redes noche. En esta evaluación se obtuvo un total de 1280 trampas noche y 41 redes noche. Los esfuerzos por cada localidad se presentan en la Tabla 2.

La diversidad de los lugares de muestreo fue estimada con los índices de diversidad de Shannon-Wiener y el índice de Simpson (Krebs, 1999), comparándose también la riqueza de especies y la equitatividad.

El estado de conservación de las especies, especialmente las endémicas y amenazadas, son evaluadas con el contexto de la legislación vigente (Decreto Supremo No 034-2004-AG), y a los acuerdos internacionales (CITES 2006, IUCN 2006). Finalmente, se sugiere algunas iniciativas de conservación de la cuenca del río Apurímac.

\section{Resultados}

\section{Composición de especies}

Sesenta especies de mamíferos son registradas para las cinco localidades evaluadas en la cuenca del río Apurímac, las que incluyen dos didelfimorfios, un cingulado, cuatro primates, 17 roedores, 23 murciélagos, ocho carnívoros, un perisodáctilo y cuatro artiodáctilos (Tabla 3).

Como era de esperarse en el área de estudio, los murciélagos (23 especies) y los roedores (17 especies) fueron los órdenes más diversos, sumando ambos el $66,7 \%$ del total de especies, mientras que los otros seis órdenes reportados acumulan solo el 33,3\% de especies. Dentro de estos órdenes, las familias Phyllostomidae y Cricetidae fueron las más representadas $(33,3$ y $18,3 \%$ respectivamente). Los cingulados (una especie) y perisodáctilos (una especie) fueron los órdenes menos diversos. Por otro lado, a nivel de familias, los roedores y los carnívoros presentaron la mayor diversidad, con seis y cinco familias respectivamente.

Al compararse las localidades (Tabla 3, Fig. 2), se observa que Catarata y Ccentabamba, ambas a menor elevación y en bosque premontano, registraron la mayor diversidad de especies con 27 y 24 especies respectivamente, mientras que Cconoc fue el lugar menos diverso (siete especies). A nivel de familias, Ccentabamba es notablemente más diverso, pero a nivel de órdenes todos los lugares con excepción de Cconoc presentan una diversidad similar (Tabla 3). Cconoc, con un hábitat de bosque seco interandino, es la localidad menos diversa en todas las categorías taxonómicas. Con excepción de esta localidad, el número de especies disminuye mostrando una correlación inversa significativa $(P<0,05)$ con los cambios de elevación $(r=0,99)$.

Se observa también que Velavelayoc y Cconoc presentaron un alto número de especies de carnívoros y pocas especies de mamíferos pequeños, a diferencia de Ccentabamba y Catarata (bosque premontano) y Yanamonte (bosque montano) donde los murciélagos y roedores son los más diversos respectivamente(Fig. 2).

Entre las especies registradas, varios son nuevos registros regionales o proporcionan información adicional sobre la especie. Se resalta al marsupial Monodelphis peruviana, capturado en Yanamonte, por ser el primer registro para el departamento de Ayacucho y el más alto conocido para la especie (ver Solari et al. 2006); el roedor Thomasomys cf. kalinowskii, capturado también en Yanamonte, por que podría representar una especie nueva semejante a T. kalinowskii; el murciélago Artibeus planirostris, colectado en Cconoc, es el primer registro para el departamento de Apurímac, el registro más alto $(1820 \mathrm{~m})$ y posiblemente el primer registro para bosque seco interandino (ver Koopman, 1978; Solari et al., 2006); el murciélago Lonchophylla handleyi, raro en otros bosques premontanos, es localmente abundante en Catarata, probablemente por la presencia en este lugar de grandes riscos apropiados para refugios; el murciélago Myotis keaysi, colectado en Velavelayoc, es el primer registro para el departamento de Apurímac (ver LaVal 1973); el venado enano Mazama chunyi, registrada por una piel y entrevistas en Yanamonte, es el

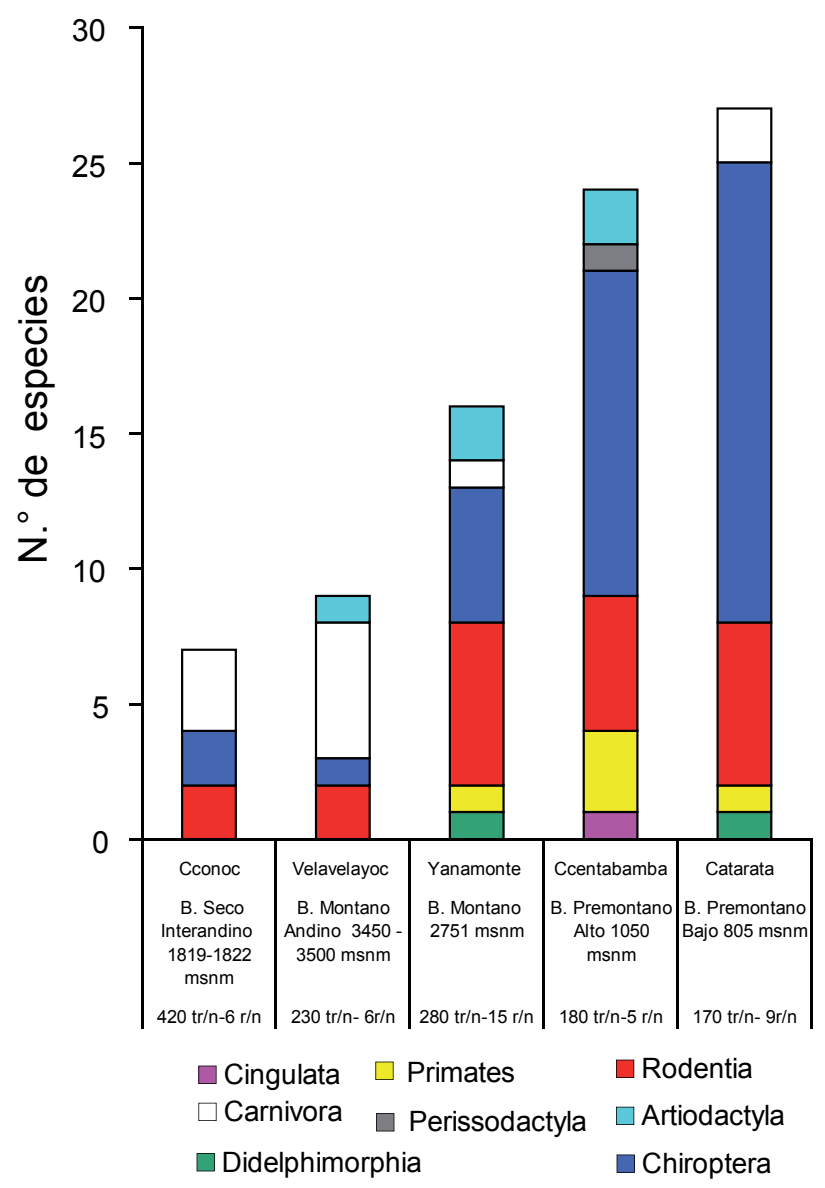

Figura 2. Número de especies de mamíferos registradas por localidad en la cuenca del río Apurímac. Se considera el total de registros por captura (tr/n =trampas noche, $r / n=r e d e s$ noche), encuesta, avistamiento, huellas, heces y otros. 
Tabla 3. Lista de especies de mamíferos registrados en la cuenca del río Apurímac.

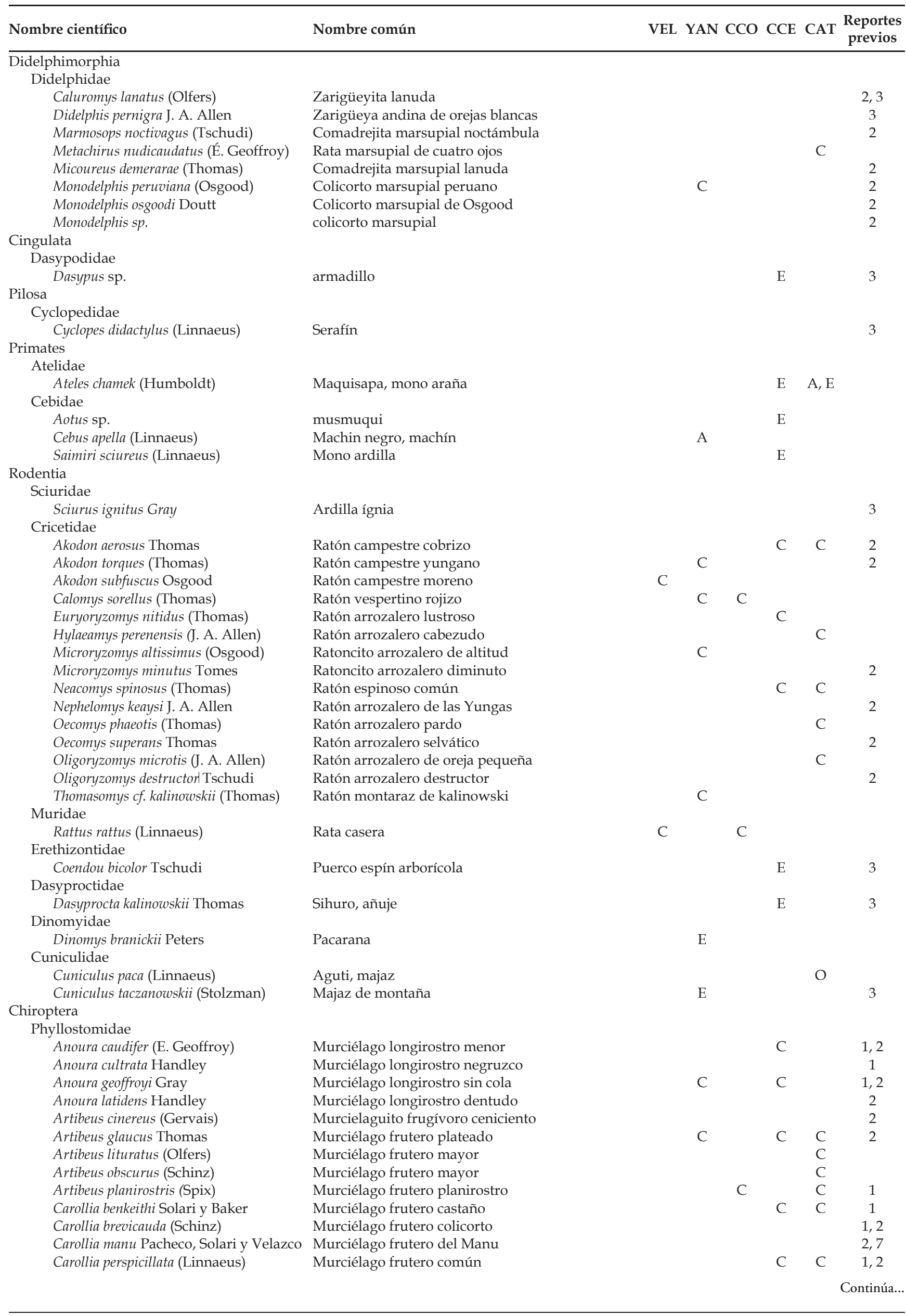


Tabla 3. ...

Chiroderma villosum Peters Chiroderma trinitatum Goodwin Desmodus rotundus (E. Geoffroy) Enchistenes hartii (Thomas) Glossophaga soricina (Pallas) Lonchophylla handleyi Hill Mesophylla macconnelli Thomas Mimon koepckeae Gardner y Patton Phyllostomus hastatus (Pallas) Platyrrhinus albericoi Velazco Platyrrhinus helleri (Peters) Platyrrhinus infuscus (Peters) Platyrrhinus masu Velazco Platyrrhinus nigellus (Gardner y Carter) Sturnira aratathomasi Peterson yTamsitt Sturnira bidens Thomas Sturnira erythromos (Tschudi) Sturnira lilium (E. Geoffroy) Sturnira magna de la Torre Sturnira nana Gardner y O'Neill Sturnira oporaphilum (Tschudi) Sturnira tildae de la Torre Uroderma bilobatum Peters Vampyressa bidens (Dobson) Vampyrodes caraccioli (Thomas) Vespertilionidae

Eptesicus brasiliensis (Desmarest) Myotis keaysi J. A. Allen Myotis nigricans (Schinz) Carnivora Myotis riparius Handley

Felidae

Leopardus pardalis (Linnaeus)

Leopardus tigrinus Schreber

Puma concolor (Linnaeus)

Puma yagouaroundi (É. Geoffroy SaintHilaire)

Canidae

$$
\text { Lycalopex culpaeus (Molina) }
$$

Ursidae

Tremarctos ornatus (F. G. Cuvier)

Mustelidae

Conepatus chinga (Molina)

Eira barbara (Linnaeus)

Mustela frenata Lichtenstein

Lontra longicaudis (Olfers)

Procyonidae

Bassaricyon alleni Thomas

Nasua nasua (Linnaeus)

Nasuella sp.

Potos flavus (Schreber)

Perissodactyla

Tapiridae

Tapirus terrestris (Roulin)

Artiodactyla

Tayassuidae

$$
\text { Pecari tacaju (Linnaeus) }
$$

Cervidae

Mazama americana (Erxleben)

Mazama chunyi Hershkovitz

Odocoileus peruvianus (Gray)
Murciélago de lineas tenues

Murciélago menor de listas

Vampiro común

Murciélago frutero aterciopelado

Murciélago longirostro de Pallas

Murciélago longirostro de Handley

Murcielaguito cremoso

Murciélago de hoja nasal peluda de Koepcke

Murciélago hoja de lanza

Muciélago de listas de Alberico

Murciélago de listas claro

Muciélago de listas tenues

Muciélago de listas masu

Murciélago de listas peruano

Murciélago de hombros amarillos de Aratathomas

Murciélago de hombros amarillos de dos dientes

Murciélago frugívoro oscuro

Murciélago de charreteras amarillas

Murciélago de hombros amarillos grande

Murciélago frugívoro enano

Murciélago de charreteras del oriente

Murciélago de charreteras rojizas

Murciélago constructor de toldos

Murcielaguito de lista dorsal

Murciélago de listas pronunciadas

Murciélago parduzco

Murciélago negruzco

Murciélago negruzco común

Murcielaguito acanelado

C

Tigrillo

Gato tigre común, tigrino

Puma

Yahuarundi, matsonsori

Zorro colorado

E

E E

E

Oso de anteojos

E

E

Tejón, manco

Comadreja

Nutria, mayopuma

E

Olingo, chosna

Coatí de cola anillada

Capiso

Chosna

Sachavaca

Sajino

E

Venado colorado

E

O, E 3

E 3

3

C C

6

1

1,2

C $\mathrm{C} \quad 1,2$

1,2

1,2
1,2

C 1

C C 1, 2

C 1

C

C 1,2

1

2

Venado enano

E, P

Venado cola blanca

\begin{tabular}{|c|c|c|c|c|c|}
\hline Total Ordenes & 4 & 6 & 3 & 6 & 5 \\
\hline Total Familias & 8 & 8 & 5 & 12 & 6 \\
\hline Total Especies & 9 & 16 & 7 & 24 & 27 \\
\hline
\end{tabular}
E E

Localidades: $\mathrm{VEL}=$ Velavelayoc, YAN $=$ Yanamonte, $\mathrm{CCO}=$ Cconoc, $\mathrm{CCE}=$ Centambamba, CAT = Catarata .

Tipo de registro: $A=$ avistamientos cerca al lugar de muestreo, $C=$ captura, $E=$ entrevistas, $P=p i e l, O=$ otros.

Reportes previos: 1= Romo (2001), 2= Solari et al. (2001a), 3= Rodríguez y Amanzo (2001), 4= Pacheco y Hocking (2006), 5 = Gardner y O’Neill. (1971), 6 = Gardner y Patton (1972), 7 = Pacheco et al. (2004). 
Tabla 4. Abundancia relativa de mamíferos terrestres no voladores por localidad evaluada en la cuenca del río Apurímac.

\begin{tabular}{|c|c|c|c|c|c|}
\hline 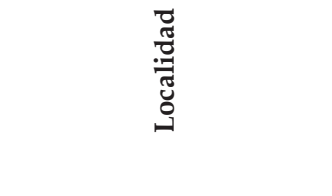 & $\begin{array}{l}\text { d } \\
\frac{3}{0} \\
\frac{0}{0} \\
>\end{array}$ & 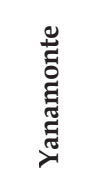 & $\begin{array}{l}\mathscr{0} \\
\tilde{U} \\
\tilde{U}\end{array}$ & 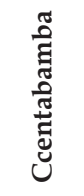 & 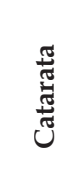 \\
\hline Akodon aerosus & & & & 1,67 & 4,7 \\
\hline Akodon subfuscus & 1,74 & & & & \\
\hline Akodon torques & & 11,07 & & & \\
\hline Calomys sorellus & & 0,36 & 0,24 & & \\
\hline Euryoryzomys nitidus & & & & 1,67 & \\
\hline Hylaeamys perenensis & & & & & 4,12 \\
\hline Metachirus nudicaudatus & & & & & 0,59 \\
\hline Microryzomys altissimus & & 0,36 & & & \\
\hline Monodelphis peruviana & & 0,36 & & & \\
\hline Neacomys spinosus & & & & 0,56 & 0,59 \\
\hline Oecomys phaeotis & & & & & 0,59 \\
\hline Oligoryzomys microtis & & & & & 1,18 \\
\hline Thomasomys cf. kalinowskii & & 3,93 & & & \\
\hline Rattus rattus & 0,83 & & 1,43 & & \\
\hline Total & 2,57 & 16,08 & 1,67 & 3,90 & 11,77 \\
\hline
\end{tabular}

primer registro para el departamento de Ayacucho, el registro más occidental conocido para la especie y el primer registro en la margen izquierda del río Apurímac (ver Grimwood, 1969; Rumiz et al., 2006); el mayopuma o nutria Lontra longicaudis es el primer registro para el departamento de Apurímac, registrada por entrevistas en Cconoc (ver Larivière, 1999; Patterson et al., 2005). El capiso Nasuella sp., un coatí de montaña, fue registrada por encuestas en Velavelayoc, coincidiendo en la descripción con otros especímenes que son tema de otra publicación (Pacheco V., y P. Hocking, com. pers.).

\section{Abundancia relativa}

Se obtuvo datos de abundancia relativa (AR) para las especies capturadas y se analizan para el grupo de mamíferos no voladores (roedores, marsupiales) y voladores (murciélagos). En el primer grupo se observa que la localidad de Yanamonte registra la mayor abundancia seguida de Catarata, indicando que la AR obtenida no muestra correlación con la elevación $(r=-0,05)$. La AR tampoco parece estar relacionado al tipo de hábitat, dado que Ccentabamba y Catarata tienen hábitats similares y ambos presentan amplias diferencias en AR. Cconoc registra los valores de AR más bajos, y es preocupante encontrar que Rattus rattus fue el roedor más abundante. En el bosque montano de Velavelayoc y especialmente en Yanamonte, las especies de Akodon alcanzaron la más alta $\mathrm{AR}$, mientras que a menores elevaciones en Ccentabamba y Catarata Akodon aerosus, Euryoryzomys nitidus y Hylaeamys perenensis fueron los más abundantes. Thomasomys cf. kalinowskii fue la segunda especie más abundante en Yanamonte. Los didelfimorfios Metachirus nudicaudatus y Monodelphis peruviana fueron más bien raros, con AR muy bajos (Tabla 4).

Para los murciélagos, la abundancia relativa es notablemente más alta a menores elevaciones (Tabla 5), mostrando una correlación inversa significativa $(P<0,05)$ con la elevación $(r=-0,91)$.
Cconoc es nuevamente preocupante por la alta abundancia del vampiro Desmodus rotundus. En el bosque montano de Yanamonte, Sturnira erythromos fue la especie más abundante, mientras que Carollia perspicillata fue la más abundante en Ccentabamba y segunda en Catarata. Lonchophylla handleyi fue la especie más abundante en Catarata, en contraste con otros lugares donde las especies de Lonchophylla son generalmente raras (Woodman y Timm, 2006).

Los registros realizados por avistamientos, encuestas, huellas, heces, etc. nos permiten presentar un estimado cualitativo de abundancia. En el departamento de Apurímac registramos que el oso de anteojos Tremarctos ornatus fue, 10 años atrás, frecuente en todo el distrito de Huanipaca; sin embargo, ahora se le conoce sólo en las localidades de Ejnay, Carquei y San Ignacio. En Velavelayoc se han realizado avistamientos recientes por pobladores locales y se conoce que de allí provienen un par de oseznos actualmente en exhibición en el Parque Zoológico de Abancay, por lo que es considerado frecuente. De igual forma, el venado de cola blanca Odocoileus peruvianus, el zorro Lycalopex culpaeus, el zorrillo Conepatus chinga, la comadreja Mustela frenata y el capiso Nasuella sp. son consideradas especies frecuentes en la zona. De esta última especie, un poblador mencionó que el

Tabla 5. Abundancia relativa de las especies de murciélagos por localidad evaluada en la cuenca del río Apurímac.

\begin{tabular}{|c|c|c|c|c|c|}
\hline 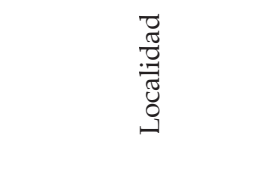 & 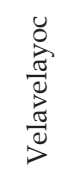 & 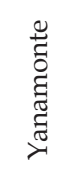 & 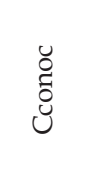 & 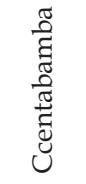 & 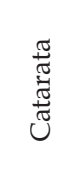 \\
\hline Anoura caudifer & & & & 2,00 & \\
\hline Anoura geoffroyi & & 1,33 & & 2,00 & \\
\hline Artibeus glaucus & & 1,33 & & 2,00 & 4,44 \\
\hline Artibeus lituratus & & & & & 2,22 \\
\hline Artibeus obscurus & & & & & 1,11 \\
\hline Artibeus planirostris & & & 20,00 & & 1,11 \\
\hline Carollia benkeithi & & & & 2,00 & 1,11 \\
\hline Carollia perspicillata & & & & 52,00 & 11,11 \\
\hline Chiroderma trinitatum & & & & & 1,11 \\
\hline Desmodus rotundus & & 0,67 & 10,00 & & \\
\hline Eptesicus brasiliensis & & & & & 1,11 \\
\hline Glossophaga soricina & & & & 2,00 & 2,22 \\
\hline Lonchophylla handleyi & & & & 2,00 & 21,11 \\
\hline Myotis keaysi & 2,50 & & & & 1,11 \\
\hline Myotis nigricans & & & & 2,00 & \\
\hline Phyllostomus hastatus & & & & & 1,11 \\
\hline Platyrrhinus helleri & & & & 2,00 & 2,22 \\
\hline Platyrrhinus nigellus & & & & 2,00 & 1,11 \\
\hline Sturnira erythromos & & 5,33 & & & \\
\hline Sturnira lilium & & & & 6,00 & \\
\hline Sturnira oporaphilum & & 0,67 & & 8,00 & 2,22 \\
\hline Uroderma bilobatum & & & & & 2,22 \\
\hline Vampyrodes caraccioli & & & & & 1,11 \\
\hline Total & 2,50 & 9,33 & 30,00 & 84,00 & 57,78 \\
\hline
\end{tabular}


capiso forma grupos de 25-30 individuos, en parejas y/o solitarios y que se le ve comiendo raíces durante el día y son fácilmente visibles en julio-agosto.

En Cconoc, la nutria o mayopuma Lontra longicaudis es vista a orillas del río Apurímac, pero es considerada rara en la zona. En contraste, el zorro Lycalopex culpaeus y el zorrillo Conepatus chinga son considerados comunes.

En Yanamonte, el oso de anteojos Tremarctos ornatus es considerado frecuente porque los pobladores mencionan que se aproxima a sus chacras, muchas veces causando daños. De igual forma, el venado enano Mazama chunyi y el venado de cola blanca Odocoileus peruvianus son considerados frecuentes por los pobladores locales. Un campesino, describió con precisión al majaz de montańa Cuniculus taczanowskii y la pacarana Dinomys branickii, agregando que son especies cazadas. En Ccano, poblado debajo de Yanamonte, se observó una mascota del machín negro Cebus apella, aunque probablemente provenga de zonas más bajas y cercanas al río Apurímac donde está presente.

En los alrededores de Ccentabamba, algunos pobladores manifestaron que en las partes altas se observa, pero raramente, un armadillo Dasypus sp., el maquisapa Ateles chamek, un musmuqui Aotus sp., el machín negro Cebus apella, el mono frailecito Saimiri sciureus, el puerco espín Coendou bicolor, un añuje Dasyprocta sp., la sachavaca Tapirus terrestris, el sajino Pecari tacaju y el venado colorado Mazama americana.

En los alrededores de Catarata, colindante a la Reserva Comunal Ashaninka, se observó una mascota de maquisapa Ateles chamek en la comunidad de Sampantuari, la que es considerada rara localmente. Por otro lado, los pobladores esgrimen que los felinos (pumas y tigrillos) atacan y se llevan sus animales, por lo que se ven obligado a matarlos, siendo nosotros testigos de un tigrillo recientemente cazado. Estos felinos son aquí considerados frecuentes en la zona. También se registró al majaz Cuniculus paca por el cráneo de un animal cazado.

Tabla 6. Número de especies e individuos capturados e índices de diversidad para las localidades evaluadas en la cuenca del río Apurímac.

\begin{tabular}{|c|c|c|c|c|c|}
\hline 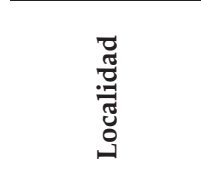 & 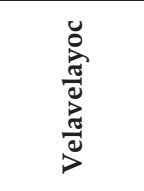 & 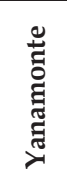 & $\begin{array}{l}\mathscr{0} \\
\text { ¿̊ }\end{array}$ & 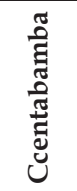 & 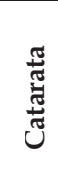 \\
\hline $\begin{array}{l}\text { Unidad de } \\
\text { vegetación }\end{array}$ & MA, BMA & $\mathrm{BM}$ & BS & $\mathrm{BPA}$ & $\mathrm{BPB}$ \\
\hline $\mathrm{N}^{\mathrm{o}}$ de especies & 3 & 10 & 4 & 15 & 23 \\
\hline $\begin{array}{c}\text { N. }{ }^{\circ} \text { de } \\
\text { individuos }\end{array}$ & 4 & 59 & 26 & 49 & 72 \\
\hline $\mathrm{H}^{\prime}$ & 1,50 & 2,16 & 1,69 & 2,55 & 3,62 \\
\hline$H^{\prime} \max$ & 1,58 & 3,32 & 2,00 & 3,91 & 4,52 \\
\hline $\mathrm{J}$ & 0,95 & 0,65 & 0,85 & 0,65 & 0,80 \\
\hline 1-D & 0,63 & 0,67 & 0,66 & 0,70 & 0,88 \\
\hline
\end{tabular}

H'= Índice de diversidad de Shannon-Wiener, H' max = Índice de diversidad máxima, $\mathrm{J}=$ Índice de heterogeneidad, 1-D= Índice de diversidad de Simpson. $B M A=$ Bosque montano andino, $M A=$ Matorral de arbustos, $\mathrm{BM}=$ Bosque montano, $\mathrm{BS}=$ Bosque seco interandino, $\mathrm{BPA}=$ Bosque premontano alto, $\mathrm{BPB}=$ Bosque premontano bajo
Tabla 7. Especies de mamíferos con su estado de conservación en la cuenca del río Apurímac.

\begin{tabular}{|c|c|c|c|c|c|}
\hline Orden & Familia & Especie & $\begin{array}{l}\text { 岩 } \\
\text { 至 } \\
\mathbf{Z}\end{array}$ & Z & $\begin{array}{l}\text { 吕 } \\
\text { 岂 }\end{array}$ \\
\hline \multicolumn{6}{|c|}{ Primates } \\
\hline & Atelidae & Ateles chamek & VU & & II \\
\hline \multirow{3}{*}{\multicolumn{2}{|c|}{ Cebidae }} & Aotus sp. & & & II \\
\hline & & Cebus apella & & & II \\
\hline & & Saimiri sciureus & & & II \\
\hline
\end{tabular}

Rodentia

$\begin{array}{llll}\text { Cricetidae } & \text { Thomasomys kalinowskii } & \text { VU } & \\ \text { Dinomyidae } & \text { Dinomys branickii } & \text { EN } & \text { EN } \\ \text { Cuniculidae } & \text { Cuniculus taczanowskii } & \text { VU } & \end{array}$

Chiroptera

Phyllostomidae Lonchophylla handleyi

VU

Carnivora

$\begin{array}{lllll}\text { Canidae } & \text { Lycalopex culpaeus } & & & \text { II } \\ \text { Mustelidae } & \text { Lontra longicaudis } & & & \text { I } \\ \text { Ursidae } & \text { Tremarctos ornatus } & \text { EN } & \text { VU } & \text { I } \\ \text { Felidae } & \text { Leopardus pardalis } & & & \text { I } \\ & \text { Puma concolor } & \text { NT } & \text { NT } & \text { II }\end{array}$

Perissodactyla

Tapiridae Tapirusterrestris VU VU II

Artiodactyla

Cervidae Mazama chunyi VU

EN: en peligro, VU: Vulnerable, NT: Cercanamente amenazado; I = Apéndice I, II = Apéndice.

Fuente: Instituto Nacional de Recursos Naturales (INRENA, 2006). Lista roja de la Unión Internacional para la Conservación de la Naturaleza y los Recursos Naturales (IUCN, 2006), Apéndices de la Convención sobre el Comercio Internacional de Especies Amenazadas de Flora y Fauna (CITES, 2006).

Aunque las encuestas se desarrollan siguiendo una metodología, estas deben ser consideradas con cautela considerándose que siempre es deseable una confirmación directa (e.g., avistamiento) de la especie.

\section{Índices de diversidad}

Los valores de los índices de diversidad de Shannon-Wiener (H') y de Simpson (1-D) obtenidos para cada localidad evaluada se presentan en la Tabla 6 . Allí se observa que Ccentabamba y Catarata son las localidades con mayor diversidad $\left(\mathrm{H}^{\prime}=2,55 \mathrm{y}\right.$ 3,62 respectivamente), mientras que Cconoc y Velavelayoc son las de menor diversidad $\left(H^{\prime}=1,69\right.$ y 1,50 respectivamente), reflejando el peso que da el índice a la riqueza de especies. En cambio con el índice de dominancia de Simpson, y con excepción de Catarata que registra el valor más alto $(1-\mathrm{D}=0,88)$, las otras localidades presentan valores bastante similares a pesar de las grandes diferencias en el número de especies. La heterogeneidad $(\mathrm{J})$ fue bastante alta en todos los lugares con excepción de Yanamonte y Ccentabamba.

Se observa que en ambientes de bosque premontano y montano (i.e., excluyendo Cconoc) hay una tendencia a encontrar una correlación inversa significativa $(P<0,05)$ entre los valores 
de H'y la elevación $(r=-0,90)$, mientras que con el índice de dominancia esta tendencia es moderada $(r=-0,80)$. No obstante, estos resultados son considerados preliminares dado que las faunas reportadas son aún incompletas.

\section{Especies protegidas por la legislación nacional e internacional.}

En las localidades evaluadas del río Apurímac se registraron 15 especies listadas en alguna categoría de amenaza en la legislación peruana (Ministerio de Agricultura, 2004), la lista roja de la Unión Internacional para la Conservación de la Naturaleza y los Recursos Naturales (IUCN 2006), o los apéndices de la Convención sobre el Comercio Internacional de Especies Amenazadas de flora y fauna (CITES 2006). Estas especies son cuatro primates, tres roedores, un murciélago, cinco carnívoros, un perisodáctilo y un venado (Tabla 7). De ellas, el oso de anteojos Tremarctos ornatus y el roedor Dinomys branickii son las especies más amenazadas; afortunadamente el oso de anteojos es aún frecuente en Velavelayoc y montañas cercanas en dirección a Choquequirao. El venado enano Mazama chunyi es también amenazado, aunque es considerado frecuente en Velavelayoc y Yanamonte. Los roedores Thomasomys cf. kalinowskii, Dinomys branickii y Cuniculus taczanowskii son considerados amenazados; sin embargo, la primera especie es considerada común localmente, mientras que las otras dos por ser cazadas podrían estar realmente amenazadas. Las especies Ateles chamek y Tapirus terrestris son consideradas raras y amenazadas en la zona. El tigrillo Leopardus pardalis es frecuente y considerado perjudicial por los pobladores por capturar las aves de corral; al parecer la presencia de animales domésticos le permite mantener una población estable a pesar de ser cazado. Desafortunadamente, no se cuenta con mayores datos para las otras especies amenazadas.

\section{Especies endémicas}

En las localidades evaluadas se registraron cuatro especies endémicas para el Perú, todos roedores pequeños: Akodon torques, Calomys sorellus, Oecomys phaeotis y Thomasomys cf. kalinowskii; los que, con excepción de $O$. phaeotis, son considerados comunes. Los roedores Akodon torques y Thomasomys cf. kalinowskii fueron registrados en bosques montanos, Calomys sorellus en bosque seco interandino y Oecomys phaeotis en bosque premontano. Lamentablemente no se obtuvo ningún registro de los murciélagos endémicos Sturnira nana y Mimon koeopcke, reportados previamente en la cuenca del Apurímac (Gardner y O’Neill, 1971; Gardner y Patton, 1972).

\section{Conservación}

La cuenca del río Apurímac (componente del VRAE) sostiene una alta diversidad de especies y hábitats en pisos ecológicos que van desde los $800 \mathrm{~m}$ hasta cerca de los $4000 \mathrm{~m}$. Lamentablemente el área presenta una alta deforestación del bosque, los que son convertidos para terrenos de agricultura, ganadería y siembra de coca. Estas actividades desarrolladas en la ausencia de una zonificación ecológica amenazan con crear amplias zonas deforestadas con la consecuente pérdida de diversidad. En la cuenca del río Apurímac no existe una política de conservación evidente ni una Unidad de Conservación de importancia con excepción del Santuario Nacional Ampay, que aunque protege bosques andinos es muy pequeño en relación al tamaño de la cuenca y no incluye bosques premontanos ni los bosques montanos del lado derecho del río Apurímac (ver Fig. 1).
El área total relativamente protegida que suman el Santuario Nacional Ampay y los sectores que pertenecen a la cuenca del río Apurímac de la Reserva Comunal Ashaninka y el Parque Nacional Otishi alcanza a $98,67 \mathrm{~km}^{2}$ aproximadamente, lo que representa tan solo el $0,36 \%$ del área de la cuenca del río Apurímac; resultado que refleja el poco esfuerzo de conservación realizado, por lo que se recomienda establecer más y más grandes áreas de protección. En este contexto, se recomienda que el Santuario Histórico Machu Picchu se amplíe hasta incluir la zona del complejo arqueológico Choquequirao, en la cabecera del río Apurímac, formando así un corredor de conservación de ambientes yunganos desde la cuenca del río Urubamba hasta la del río Apurímac.

De las zonas exploradas, tres de ellas ameritan tener algún grado de protección: 1) Huanipaca (bosque de Velavelayoc), departamento de Apurímac, es una zona importante para la protección del oso de anteojos, el coatí de montańa, y probablemente del venado enano Mazama chunyi. Además, sus montañas no están densamente pobladas y podría establecerse una unidad de conservación regional, que incluya los fragmentos de bosques desde Huanipaca hasta el mismo río Apurímac. 2) Yanamonte, departamento de Ayacucho, un impresionante bosque que cubre toda una falda del cerro, increíblemente aún bastante intacto a pesar de la agricultura de corte y quema en los alrededores. En este lugar se encuentra el venado enano Mazama chunyi y una especie nueva de roedor Thomasomys. 3) Las montańas aledańas a Catarata (Cusco) son sugeridas para conservar al tigrillo, el maquisapa y una impresionante diversidad de mamíferos pequeños. Esta es un área con alta diversidad que puede ser un centro turístico por su cercanía a poblados importantes como San Francisco.

\section{Discusión}

Desde que John W. Terborgh realizara la gran expedición a la Cordillera Vilcabamba, de 1965 a 1968, una de las más importantes del siglo veinte en el Perú, no se ha continuado con igual intensidad el estudio de la diversidad en esa zona, no obstante él advirtiera que en una o dos generaciones dichos estudios serían imposibles debido al avance de la civilización (Terborgh y Dudley, 1973). Actualmente, el valle del río Apurímac ha sido rápidamente colonizado; sin embargo, los estudios de diversidad son ahora más importantes que nunca para sustentar nuevos esfuerzos en la conservación de la zona. El presente trabajo representa por ello un esfuerzo inicial de dicho interés.

El valle del río Apurímac es ecológicamente complejo. La humedad proveniente de la selva amazónica convierte la cuenca inferior, desde el nivel del río hasta sus vertientes altas, en hábitats similares a los bosques premontanos y montanos de la vertiente oriental de los Andes. Sin embargo, a mayor elevación, a la altura de Cconoc, la cuenca alta del río Apurímac recibe menor cantidad de lluvias y son por ello secos y más similares a los hábitats de la vertiente del Pacífico que a la vertiente oriental, encontrándose zonas de bosque seco y matorral arbustivo. Similar mosaico de hábitats se encuentra sólo en otros ríos interandinos como el Marañón y el Huallaga (Koopman, 1978).

La importancia biogeográfica de la cuenca del río Apurímac se demuestra por los nuevos registros aquí obtenidos. El marsupial Monodelphis peruviana y el venado enano Mazama chunyi son primeros registros para el departamento de Ayacucho; por otro lado los murciélagos Artibeus planirostris y Myotis keaysi y la nutria Lontra longicaudis son los primeros registros para el 
departamento de Apurímac. Se confirma también la presencia del capiso Nasuella sp., un coatí de montaña, obteniéndose nuevos datos de la especie.

Como era de esperarse, Catarata y Ccentabamba (24 y 27 especies respectivamente), ambos con hábitat de bosque premontano fueron los más diversos. Por el contrario, Cconoc, es el lugar con menor riqueza de especies $(\mathrm{N}=7)$, y con los valores más bajos en los índices $\mathrm{H}^{\prime}$ y J. Estos datos sugieren que los bosques secos albergan menor diversidad de especies que los bosques tropicales (premontanos y montanos). En Cconoc, es preocupante la abundancia del vampiro Desmodus rotundus y la rata casera Rattus rattus, por estar involucradas en diversas enfermedades de importancia en la salud pública. Estas especies sugieren también un impacto antrópico en el hábitat que podría explicar también la baja diversidad encontrada.

En este reporte se confirma que, además de tener menos diversidad, el bosque seco interandino del río Apurímac presenta una fauna anidada o enrarecida de la presente en los bosques premontanos. Posiblemente, las especies premontanas desaparecen gradual e inversamente correlacionada con la elevación a lo largo del río. Sin embargo, se necesita evaluar más puntos de muestreo a lo largo del río para confirmar esta hipótesis. En forma similar, se observa que la diversidad de especies, en las vertientes con bosques montanos, disminuye con el incremento de la elevación, tal como fue documentado en la vertiente oriental (Patterson et al., 1996); y que el bosque montano presenta especies propias ausentes en los bosques premontanos (e.g., Mazama chunyi, Monodelphis peruviana, Akodon torques). Sin duda, las especies premontanas van disminuyendo también conforme aumenta la elevación, pero hacen falta más puntos de evaluación a lo largo de la gradiente para documentar este patrón.

Los resultados indican que la abundancia relativa de los mamíferos pequeños terrestres no está correlacionada con la elevación, pudiendo ser alta tanto en el bosque montano como en el premontano. En cambio, esta correlación sí es significativa para el caso de los murciélagos. Los géneros Akodon, Thomasomys y Sturnira fueron las que obtuvieron una mayor AR; similar grado de abundancia fue registrada en la Cordillera Vilcabamba (Emmons et al., 2001). Otros factores, como la cantidad de recursos disponibles y/o la competencia con otras especies podrían ser los responsables de la abundancia observada.

Tampoco es sorpresa encontrar que en la cuenca del río Apurímac, los roedores y murciélagos $(66,7 \%)$ son los grupos taxonómicos más diversos, en concordancia con lo expuesto por Pacheco (2002). Sin embargo, el que la familia Phyllostomidae sea la más numerosa, es debido a un efecto de muestreo basado más en el uso de redes. Se sugiere, en el futuro, el empleo de diversos métodos para incorporar especies de otras familias (Voss y Emmons, 1996).

En forma preliminar se concluye que la cuenca del río Apurímac alberga al menos 97 especies de mamíferos, que resulta de incluir las 60 especies aquí registradas, 28 especies listadas por Solari et al. (2001a) para las localidades de Llactahuaman y Wayrapata, 19 especies de mamíferos medianos y grandes para estas últimas localidades (Rodríguez y Amanzo (2001), 42 especies listadas para la cordillera Vilcabamba (Koopman, 1978; Romo, 2001; Solari et al., 2001a), los murciélagos Sturnira nana y Mimon koepckeae descritos para la localidad tipo de Huanhua- chayo (Gardner y O’Neill, 1971; Gardner y Patton 1972 respectivamente), el murciélago Carollia manu descrito recientemente (Pacheco et al. 2004), y el reporte de Sturnira aratathomasi para Cconoc (Pacheco y Hocking, 2006) (ver Tabla 3).

Se observa que la diversidad del río Apurímac (97 especies) es mayor a las 58 especies reportadas para la Cordillera de Vilcabamba (Emmons et al., 2001), pero es menor a las 130 especies presentes en un rango de 800-3500 m en la Reserva de Biósfera del Manu (ver Solari et al., 2006). Sin embargo, estos estimados son aún preliminares debido a que estos lugares, con excepción de la RB del Manu, están lejos de considerarse bien muestreados, por lo que una comparación con otros valles es aún prematuro.

Una parte de la cuenca del río Apurímac fue identificada como una área prioritaria de conservación, llamada Zona Prioritaria Vilcanota (Rodríguez, 1996; Rodríguez y Young, 2000); pero, en este importante trabajo las Zonas Prioritarias no fueron claramente definidas ni en la extensión geográfica ni en la diversidad que contenían, limitando la utilidad de las mismas. La ZP Vilcanota es entendida aquí como la zona que incluye los bosques premontanos y montanos de las cuencas de los ríos UrubambaVilcanota y el Apurímac; de esta forma protegería la diversidad expuesta en este trabajo, la presente en el Santuario Histórico Machu Picchu, y el Santuario Nacional Ampay. Esta propuesta es congruente con una zona definida de alta importancia para la protección de aves (Fjeldså et al., 2005).

Tal como Terborgh y Dudley (1973) anticiparan, el valle del río Apurímac ha sido ampliamente colonizado; preocupando más el extensivo uso de las áreas deforestadas dedicadas al cultivo de la coca Erythroxylum coca. Young (1996) reportó que la deforestación atribuible a la coca puede llegar tanto como a un millón de hectáreas de bosques premontanos en el Perú; pero aunque no existen datos actuales, es evidente que esta cantidad está en aumento. Fjeldså et al. (2005) identificaron la zona del río Apurímac como una de las zonas donde los cultivos de coca son la mayor preocupación para la conservación de las aves endémicas de las cordilleras Vilcanota y Vilcabamba. Este escenario, aunado al imperceptible esfuerzo de conservación en la zona, amenaza el alto nivel de endemismo y diversidad del río Apurímac.

En conclusión, la alta diversidad de mamíferos del río Apurímac, la presencia de especies únicas, mencionadas anteriormente, y la importancia biogeográfica de la zona incentivan a incrementar los estudios en la zona y demandan una mayor atención en la conservación de sus bosques, especialmente en el escenario actual de evidente deforestación.

\section{Agradecimientos}

Al INRENA por facilitarnos los permisos de colecta correspondientes. A Richard Cadenillas y Carlos Tello por su apoyo en la identificación de murciélagos. A Sergio Solari y Javier Barrio por confirmar la identificación del Monodelphis y Mazama respectivamente. Igualmente agradecemos a Sandra Velazco, Richard Cadenillas, Dyana La Rosa y dos réferis anónimos por revisar y comentar borradores del presente trabajo.

Esta investigación fue financiada gracias a fondos obtenidos a través de la Iniciativa de Especies Amenazadas (IEA) que $\mathrm{Cl}$ desarrolla en cooperación con socios locales de los países que conforman el Centro de Conservación de la Biodiversidad de los Andes Tropicales (CBC-Andes). En el Perú el Programa se desarrolla a través de las "Becas María Koepcke" coordinado por 
Conservación Internacional-CI Perú y la Asociación Peruana para la Conservación de la Naturaleza- APECO.

\section{Literatura citada}

CITES 2006. Ápéndices I, II y III. <url:http://www.cites.org/eng /app/appendices.html/.> Acceso 16/11/2006.

Emmons, L. H. \& F. Feer. 1997. Neotropical rainforest mammals. A field guide. Segunda edición. Chicago, The University of Chicago Press.

Emmons, L., L. Luna \& M. Romo. 2001. Mammals of the northern Vilcabamba mountain range, Peru. In: L. Alonso, A. Alonso, T. Schulenberg y F. Dallmeier,(Eds.). Biological and social assessments of the Cordillera de Vilcabamba, Peru. RAP Working Papers $12 \&$ SI/MAB Series 6. Conservation International, Washington, D.C. Pp. 105-109.

Fjeldså J., M.D. Álvarez, J.M. Lazcano \& B. León. 2005. Illicit crops and armed conflict as constraints on biodiversity conservation in the Andes region. Ambio 34: 205-211.

Gardner, A.L. \& J.P. O'Neill. 1971. A new species of Sturnira (Chiroptera:Phyllostomidae) from Peru. Occasional Papers of the Museum of Zoology, Louisiana State University 42:1-7.

Gardner, A.L. \& J.L. Patton. 1972. New species of Philander (Marsupialia: Didelphidae) and Mimon (Chiroptera: Phyllostomidae) from Peru. Occasional Papers of the Museum of Zoology, Louisiana State University 43:1-12.

Grimwood, I. R. 1969. The distribution and status of some Peruvian mammals. American Committee for International Wild Life protection and New York Zoological Society. Special Publication 21:1-86.

IUCN 2006. 2006 IUCN Red List of Threatened Species. < http:// www.iucnredlist.org>. Acceso 16/11/2006.

Jones, C., W. McShea, M. Conroy \& T. Kunz. 1996. Capturing Mammals. Pp. 115-155 in Measuring and Monitoring Biological Diversity - Standard Methods for Mammals (D. Wilson et al, eds). Smithsonian Institution Press. Washington and London.

Koopman, K. F. 1978. Zoogeography of Peruvian bats with emphasis on the role of the Andes. American Museum Novitates, 2651:1-33.

Krebs, C. 1999. Ecological Methodology - Segunda Edición. University of British Columbia. Canada.

Larivière, S. 1999. Lontra longicaudis. Mammalian Species 609: $1-5$.

LaVal, R.K. 1973. A Revision of the Neotropical Bats of the genus Myotis. Science Bulletin, Natural History Museum of Los Angeles County 15:1-45.

Lim B. K., M. D. Engstrom, T. E. Lee, J. C. Patton \& J.W. Bickham 2004. Molecular differentiation of large species of fruit-eating bats (Artibeus) and phylogenetic relationships based on the cytochrome $b$ gene. Acta chiropterologica 6 (1): 1-12.

Ministerio de Agricultura. 2004. Decreto Supremo No. 034-2004-AG. El Peruano Pp. 276853-276855.

Molina, M., \& J. Molinari. 1999. Taxonomy of Venezuelan whitetailed deer (Odocoileus, Cervidae, Mammalia), based on cranial and mandibular traits. Canadian Journal of Zoology 77: 632-645.

Pacheco, V. 2002. Mamíferos del Perú. In: G. Ceballos y J. Simonetti, Eds. Diversidad y conservación de los mamíferos neotropicales. CONABIO-UNAM, México D.F. Pp. 503-550.

Pacheco, V. 2003. Phylogenetic analyses of the Thomasomyini (Muroidea: Sigmodontinae) based on morphological data. $\mathrm{Ph}$ D. dissertation. The City University New York, New York, USA.

Pacheco, V. \& P. Hocking. 2006. Notably range extension of Sturnira aratathomasi Peterson and Tamsitt 1969 in Perú. Acta Chiropterologica 8 (2): 561-566.
Pacheco, V., S. Solari \& P. M. Velazco. 2004. A New Species of Carollia (Chiroptera: Phyllostomidae) from the Andes of Peru and Bolivia. Occasional Papers, Museum of Texas Tech University 236: 1-15.

Pacheco, V., H. de Macedo, E. Vivar, C. Ascorra, R. Arana-Cardó \& S. Solari. 1995. Lista anotada de los mamíferos Peruanos. Occasional Papers in Conservation Biology, 2: 1-35.

Patterson, B. D., V. Pacheco \& S. Solari. 1996. Distributions of bats along an elevational gradient in the Andes of south-eastern Peru. Journal of Zoology 240: 637-658.

Patterson B. D., G. Ceballos, W. Sechrest, M. F. Tognelli, T. Brooks, L. Luna, P. Ortega, I. Salazar \& B. E. Young. 2005. Digital distribution maps of the mammals of the western hemisphere, version 2.0. NatureServe, Arlington, Virginia.

Peyton, B. 1980. Ecology, distribution, and food habits of spectacled bears Tremarctos ornatus, in Peru. Journal of Mammalogy 61: 639-652.

Rodríguez, J.J. \& J.M.Amanzo. 2001. Medium and large mammals of the southern Vilcabamba region, Perú. In: L. Alonso, A. Alonso, T. Schulenberg y F. Dallmeier, Eds. Biological and social assessments of the Cordillera de Vilcabamba, Peru. RAP Working Papers $12 \&$ SI/MAB Series 6. Conservation International, Washington, D.C. Pp. 117-126.

Rodríguez, L. O. (Ed.). 1996. Diversidad Biológica del Perú, Zonas prioritarias para su conservación. FANPE-GTZ, INRENA. Lima.

Rodríguez L.O. \& K.R. Young. 2000. Biological Diversity of Peru: Determining Priority Areas for Conservation. Ambio 29(6): 329-337.

Romo, M. 2001. Bat species collected by Terborgh and Weske on an elevational transect of the Cordillera de Vilcabamba, Peru. In: L. Alonso, A. Alonso, T. Schulenberg y F. Dallmeier, Eds. Biological and social assessments of the Cordillera de Vilcabamba, Peru. RAP Working Papers 12 \& SI/MAB Series 6. Conservation International, Washington, D.C. P. 258

Rumiz, D. I., E. Pardo, C. F. Eulert, R. Arispe, R. B.Wallace, H. Gómez \& B. Ríos-Uzeda. 2006. New records and a status assessment of a rare dwarf brocket deer from the montane forests of Bolivia. Journal of Zoology 271(4): 428-436.

Solari, S. 2007. New species of Monodelphis (Didelphimorphia: Didelphidae) from Peru, with notes on M. adusta (Thomas, 1897). Journal of Mammalogy 88(2):319-329.

Solari, S. \& R.J. Baker. 2006. Mitochondrial DNA sequence, karyotypic, and morphological variation in the Carollia castanea Species Complex (Chiroptera: Phyllostomidae), with description of a new species. Occasional Papers, Museum of Texas Tech University 254:1-16.

Solari, S., E. Vivar, P. Velazco \& J. J. Rodríguez. 2001a. Small mammal diversity from several montane forest localities (1300-2800 m) on the eastern slope of the Peruvian Andes. In: L. Alonso, A. Alonso, T. Schulenberg and F. Dallmeier, Eds. Biological and social assessments of the Cordillera de Vilcabamba, Peru. RAP Working Papers 12 \& SI/MAB Series 6. Conservation International, Washington D.C. Pp. 262-264.

Solari, S., E. Vivar, P. Velazco \& J. J. Rodríguez. 2001b. Small mammals of the southern Vilcabamba region, Peru. In: L. Alonso, A. Alonso, T. Schulenberg and F. Dallmeier, Eds. Biological and social assessments of the Cordillera de Vilcabamba, Peru. RAP Working Papers 12 \& SI/MAB Series 6. Conservation International, Washington D.C. Pp. 110-116.

Solari, S., V. Pacheco, L. Luna, P.M. Velazco \& B.D. Patterson. 2006. Mammals of the Manu Biosphere Reserve, pp. 13-22. In: B.D. Patterson, D.F. Stotz, and S. Solari (Eds.) Mammals and Birds of the Manu Biosphere Reserve, Peru. Fieldiana: Zoology, new series 110 . 
Terborgh, J. \& T. R. Dudley. 1973. Biological exploration of the northern Cordillera Vilcabamba, Peru. National Geographic Society, Research Reports, 1966 projects: 255-264.

Velazco, P.M. 2005. Morphological Phylogeny of the bat genus Platyrrhinus Saussure, 1860 (Chiroptera:Phyllostomidae) with the description of four new species. Fieldiana: Zoology, new series, 105: 1-53.

Voss, R. \& L. Emmons. 1996. Mammalian diversity in neotropical lowland rainforest: A preliminary assessment. Bulletin of the American Museum of Natural History 230:1-115.

Weksler, M., A.R. Percequillo \& R.S. Voss. 2006. Ten new genera of oryzomyine rodents (Cricetidae: Sigmodontinae). American Museum Novitates 3537:1-29.

Wilson, D. E. \& D. M. Reeder (Eds.). 2005. Mammal Species of the World, Tercera Edición. Johns Hopkins University Press,
Baltimore, MD.

Woodman N., R. M. Timm, N. A. Slade \& T. J. Doonan. 1996. Comparison of traps and baits for censusing small mammals in Neotropical lowlands. Journal of Mammalogy 77 : 274-281.

Woodman, N. \& R. M. Timm. 2006. Characters and phylogenetic relationships of nectar-feeding bats, with descriptions of new Lonchophylla from western South America (Mammalia: Chiroptera: Phyllostomidae: Lonchophyllini). Proceedings of the Biological Society of Washington 119(4): 437-476.

Young K. R. 1996. Threats to biological diversity caused by coca/ cocaine deforestation in Peru. Environmental Conservation 23: 7-15. 